\title{
COVID-19 Epidemic Compartments Model and Bangladesh
}

\author{
Md. Shahidul Islam ${ }^{1}$, Jannatun Irana Ira ${ }^{2}$, K. M. Ariful Kabir ${ }^{3}$, and Md. Kamrujjaman*4,5 \\ 1,2,4 Department of Mathematics, University of Dhaka, Dhaka 1000, Bangladesh \\ ${ }^{3}$ Interdisciplinary Graduate School of Engineering Sciences, Kyushu University, Japan \\ ${ }^{5}$ Department of Mathematics and Statistics, University of Calgary, Calgary, AB, Canada \\ ${ }^{1}$ E-mail: mshahidul11@yahoo.com \\ ${ }^{2}$ E-mail: zannatunirana@gmail.com \\ ${ }^{3}$ E-mail: k.ariful@yahoo.com \\ ${ }^{4,5}$ E-mail: kamrujjaman@du.ac.bd
}

\begin{abstract}
:
In the promptness of the COVID-19 outbreak, it would be very important to observe and estimate the pattern of diseases to reduce the contagious infection. To study this effect, we developed a COVID-19 epidemic model that incorporates five various groups of individuals. Then we analyze the model by evaluating the equilibrium points and analyzing their stability as well as determining the basic reproduction number. Also, numerical simulations show the dynamics of a different group of the population over time. Thus, our findings based on the sensitivity analysis and the reproduction number highlight the role of outbreak of the virus that can be useful to avoid a massive collapse in Bangladesh and rest of the world. The outcome of this study concludes that outbreak will be in control which ensure the social and economic stability.

Keywords: Coronavirus; epidemic model; global pandemic; COVID-19; SEII $_{s} \mathrm{R}$ model; sensitivity analysis.
\end{abstract}

\section{Introduction}

Coronavirus disease 2019, which is also known as COVID-19, is an infectious disease which causes respiratory syndrome. It was first originated on 31st December 2019, in Wuhan city, Hubei Province of China. Later on 7th January 2020, it was confirmed that the disease is caused by a new kind of coronavirus. Outside of China the same kind of disease were reported in Thailand, Japan and South Korea in the same month. As the number of cases were increasing at a high rate throughout the world, the World Health Organization

${ }^{*}$ Corresponding author: M. Kamrujjaman (email: kamrujjaman@du.ac.bd) 
(WHO) declared the disease as a pandemic on March 11, 2020 [1]. This new kind of coronavirus disease is more transmissible from human-to-human. The virus was named SARS-CoV-2 by WHO [2]. Since then, 209 countries and territories have reported to have coronavirus patients [3].

\section{Background}

The coronavirus is a zoonotic disease that come from animals to humans [2]. It is a droplet infection which means it transmits from an infected persons droplet of cough or sneeze. The infected person may suffer from symptoms like fever, cough, flu, tiredness, soar throat, shortness of breath, body aches and other symptoms. In severe cases it causes pneumonia. In most of the cases, the symptoms are reported mild among the patients and these mild cases may not even require any serious level of treatment. But the patients with other diseases like diabetes, heart disease, asthma etc. faces more critical illness [1].

According to WHO more than 1.6 million people has been infected by the virus and almost 0.1 million people has been reported dead till 10 April 2020 due to the infection $[1,4]$. Nearly 82 thousands people are affected in China and the outbreak has made the Wuhan city stay locked down for more than two months. Outside of China, USA, Italy, Iran, Spain, France and UK have seen most cases and USA is currently the most affected country with more than 0.5 million patients and more than 17 thousands deaths which is still increasing rapidly. Meanwhile Italy has already 18 thousands death reports [4]. The number of cases is still growing exponentially. This dynamics of growth in population can be described by Malthusian and logistic growth curve and these models are very useful to predict the situation of such an outbreak [3].

Due to the severity, as a protection most of the government of the countries has locked everything down and they are encouraging people to stay at home and avoid public gathering. Experts are suggesting the social distancing to flatten the curve of new cases. As a result, right now more than $1 / 3$ global population is lockdown. The WHO is also spreading awareness and advising people to stay at their own habitat without emergency. Meanwhile providing instructions on how to protect oneself from the virus and any kinds of information regarding the pandemic.

\section{Case: Bangladesh}

The global pandemic has also made an impact on the overpopulated developing country Bangladesh. In Bangladesh, the Institute of Epidemiology, Disease Control and Research (IEDCR) has reported the first 3 cases of coronavirus on 8 March 2020. Currently the country has total 424 coronavirus cases with 27 deaths (April 10, 2020). We have used these results to analyze the situation of Bangladesh through the formulated model. Unfortunately in 33 days after having the first case of the pandemic, only 7359 tests samples were collected in total [5]. Initially the country had only one coronavirus testing center for the whole country until March 30 and increased to 6 Labs on March 31 and recently the government increased 13 more centers and planning to have more facilities within 20 
April. After the increament of testing facilities the country's number of new cases has also started to grow up. Due to the lacking of the real data related to the situation, it is a challenging task to estimate the parameters accurately.

\section{Epidemic Models}

In epidemiology to analyze this kind of infectious virus compartmental modelling is widely used and it is also very effective to predict the results of an epidemic successfully. A compartmental model is consists of different groups of individuals whose characters due to a disease is described by a differential equation. Some commonly used epidemic models are SIS, SID, SIR and SEIR models. The Kermack-Mckendric model is the simplest compartmental model which is also known as the SIR model [6]. But for many of the infectious disease where infected individuals can carry the infection without showing symptoms for a while (such as, chicken pox, malaria, tuberculosis etc.) an SEIR model is mostly used [7]. This epidemic models can easily analyze the dynamics of the infectious diseases and with more compartments the real life situation can be implemented even more successfully. Therefore, the compartmental modelling can be very useful tool to predict the nature of todays most dangerous disease.

In this study, we have formulated a five compartmental model considering the situations of the disease. The framework of our study are outlined in the following lists:

1. We analyze the stability of the equilibrium points of the model and calculate the basic reproduction number to understand the severity.

2. The study consists of the sensitivity analysis of the reproduction number with respect to the parameters that we have used.

3. The sensitivity analysis gives us the idea about the relative importance of the parameters in the process of transmission of the virus.

4. By using the model, we have studied the coronavirus situation of the world as well as for Bangladesh.

5. Numerically implemented results ensure the dramatic scenarios to control the spread of COVID-19.

In the following section, we will discuss our mathematical model and formulation of the model elaborately.

\section{Mathematical Model and Formulation}

The most common compartmental model is the SIR model which has three individuals, susceptible (S), infected (I) and recovered (R). If the infected individuals grow more than 
compared to recovered individuals then it is most likely to have an outbreak of the disease. We are already coping with such an outbreak COVID-19. To model the COVID-19 pandemic, we consider such compartmental modelling with more compartments. Already many models have been formulated related to the disease transmission since the outbreak in China. A SVEIJR model with 6 compartments consisting of susceptible (S), vaccinated $(\mathrm{V})$, exposed $(\mathrm{E})$, infected $(\mathrm{I})$, isolated or quarantined $(\mathrm{J})$ and recovered $(\mathrm{R})$ population have been discussed in [8]. Also a SEIQR model with quarantined population have been analyzed in [9].

In this paper, we have formulated a modified SEIR model with 5 different groups of population considering the isolation process. The five individuals are the susceptible $(\mathrm{S})$, the exposed $(\mathrm{E})$, the infected $(\mathrm{I})$, the isolated $\left(I_{s}\right)$ and the recovered $(\mathrm{R})$. The group of people who have not been infected yet but are at the risk of getting infected are the susceptible individuals (S). Those who have already been infected but in incubation period are the exposed individuals (E). These individuals are asymptomatic and they can carry the coronavirus without showing any symptoms from 2-14 (or 2-27) days [1]. Those who are infected and symptomatic are the infected individuals (I). From these infected people those who tested positive with the virus get separated from rest of the population, so that the transmission of the virus can be restricted and these individuals are the isolated population $\left(I_{s}\right)$. Lastly when the infected and the isolated patients get cured they are recovered individuals $(\mathrm{R})$ and they will not get infected again by the virus. So the model follow the diagram below:

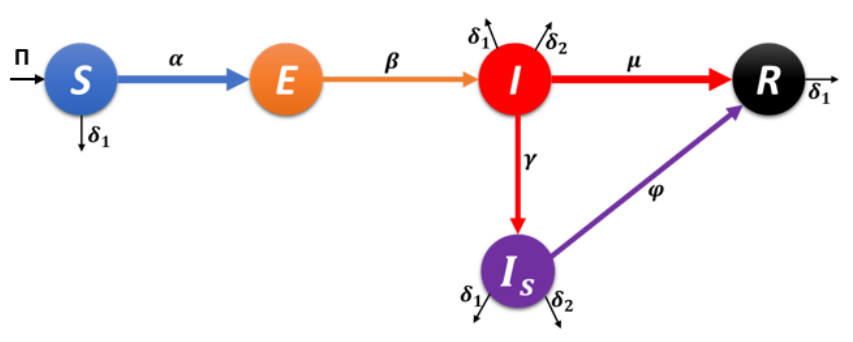

Figure 1: Diagram of the $\mathrm{SEII}_{s} \mathrm{R}$ model.

The susceptible population is getting exposed in $\alpha$ rate and $\delta_{1}$ is the natural death rate of the entire population. If we assume that none of the exposed population during the incubation period is dying then they are getting infected at $\beta$ rate. The infected individuals may die by the disease or naturally. Otherwise $\gamma I$ individuals get isolated and $\mu I$ portion of them recover from the disease and get immunity. Similarly, the isolated patients may die because of the disease or get recovered in a $\phi$ rate. Hence the model has the following 
mathematical form:

$$
\begin{cases}\frac{d S}{d t}= & \Pi-\alpha S \frac{I}{N}-\delta_{1} S \\ \frac{d E}{d t}= & \alpha S \frac{I}{N}-\beta E \\ \frac{d I}{d t}= & \beta E-\left(\gamma+\mu+\delta_{1}+\delta_{2}\right) I \\ \frac{d I_{s}}{d t}= & \gamma I-\left(\phi+\delta_{1}+\delta_{2}\right) I_{s} \\ \frac{d R}{d t}= & \phi I_{s}+\mu I-\delta_{1} R\end{cases}
$$

with the initial conditions $S(0) \geq 0, E(0) \geq 0, I(0)>0, I_{s}(0) \geq 0, R(0) \geq 0$, where,

- $\mathrm{N}$ is the total population of the region and $N=S+E+I+I_{s}+R$,

- $\Pi$ is recruitment rate of susceptible population,

- $\alpha$ is the exposure rate of the susceptible population,

- $\delta_{1}$ denotes the natural death rate of the population,

- $\beta$ is the transmission rate of exposed population to infected population,

- $\gamma$ represents the rate at which the infected population that clearly shows the symptoms of the disease are getting isolated,

- $\mu$ is the rate at which infected people that are not isolated get recovered,

- $\delta_{2}$ denotes the death rate due to the virus,

- $\phi$ is the rate at which isolated population are getting recovered.

Let us now divide all the equations of (2.1) by $\mathrm{N}$ and set, $s=\frac{S}{N}, e=\frac{E}{N}, i=\frac{I}{N}, i_{s}=\frac{I_{s}}{N}$, $r=\frac{R}{N}$ and also let the parameter $\frac{\Pi}{N}=\pi$ then (2.1) becomes

$$
\left\{\begin{array}{l}
\frac{d s}{d t}=\pi-\alpha s i-\delta_{1} s \\
\frac{d e}{d t}=\alpha s i-\beta e \\
\frac{d i}{d t}=\beta e-\left(\gamma+\mu+\delta_{1}+\delta_{2}\right) i \\
\frac{d i_{s}}{d t}=\gamma i-\left(\phi+\delta_{1}+\delta_{2}\right) i_{s} \\
\frac{d r}{d t}=\phi i_{s}+\mu i-\delta_{1} r
\end{array}\right.
$$

where, $s+e+i+i_{s}+r=1$ and the initial conditions become

$$
s(0) \geq 0, e(0) \geq 0, i(0)>0, i_{s}(0) \geq 0, r(0) \geq 0 .
$$

In the next section, we will establish some basic results that will used throughout the paper. 


\section{Auxiliary Results}

\subsection{Disease Free Equilibrium (DFE)}

The disease free equilibrium of (2.2) can be obtained easily by setting $e=i=i_{s}=r=0$. So from (2.2) we get, $\frac{d s}{d t}=0$ which gives us that, $s=\frac{\pi}{\delta_{1}}$. Therefore the DFE of (2.2) is

$$
E_{0}=\left(\frac{\pi}{\delta_{1}}, 0,0,0,0\right)
$$

\subsection{Endemic Equilibrium Point (EEP)}

To derive the endemic equilibrium point of (2.2) we set

$$
\frac{d s}{d t}=\frac{d e}{d t}=\frac{d i}{d t}=\frac{d i_{s}}{d t}=\frac{d r}{d t}=0
$$

Then we get a system of equations as follows:

$$
\begin{aligned}
& \pi-\alpha s i+\delta_{1} s=0 \\
& \alpha s i-\beta e=0 \\
& \beta e-\left(\gamma+\mu+\delta_{1}+\delta_{2}\right) i=0 \\
& \gamma i-\left(\phi+\delta_{1}+\delta_{2}\right) i_{s}=0 \\
& \phi i_{s}+\mu i-\delta_{1} r=0
\end{aligned}
$$

Solving these equations simultaneously, we get

$$
\begin{aligned}
s & =\frac{\pi}{\alpha i-\delta_{1}} \\
e & =\frac{\alpha \pi i}{\beta\left(\alpha i-\delta_{1}\right)} \\
i & =\frac{\gamma \delta_{1}+\mu \delta_{1}+\delta_{1}^{2}+\delta_{1} \delta_{2}+\alpha \pi}{\alpha\left(\gamma+\mu+\delta_{1}+\delta_{2}\right)} \\
i_{s} & =\frac{\gamma i}{\phi+\delta_{1}+\delta_{2}} \\
r & =\frac{1}{\delta_{1}}\left[\frac{\phi \gamma i}{\phi+\delta_{1}+\delta_{2}}+\mu i\right]
\end{aligned}
$$

Then setting $i=D$, we obtain the endemic equilibrium point (EEP)

$$
E_{1}=\left(\frac{\pi}{\alpha D-\delta_{1}}, \frac{\alpha \pi D}{\beta\left(\alpha D-\delta_{1}\right)}, D, \frac{\gamma D}{\phi+\delta_{1}+\delta_{2}}, \frac{1}{\delta_{1}}\left[\frac{\phi \gamma D}{\phi+\delta_{1}+\delta_{2}}+\mu D\right]\right) .
$$

It's now time to analyze the stability of the equilibrium points and to show the effects of basic reproduction number. 


\section{Stability Analysis of Equilibrium}

\subsection{Analysis of DFE}

To analyze the stability of the disease free equilibrium, let DFE is

$$
E_{0}=(x, y, z, u, v)=\left(\frac{\pi}{\delta_{1}}, 0,0,0,0\right)
$$

and then we write the Jacobian matrix of the system in $(2.2)$

$$
J=\left(\begin{array}{ccccc}
-\left(\alpha z+\delta_{1}\right) & 0 & -\alpha x & 0 & 0 \\
\alpha z & -\beta & \alpha x & 0 & 0 \\
0 & \beta & -\left(\gamma+\mu+\delta_{1}+\delta_{2}\right) & 0 & 0 \\
0 & 0 & \gamma & -\left(\phi+\delta_{1}+\delta_{2}\right) & 0 \\
0 & 0 & \mu & \phi & -\delta_{1}
\end{array}\right)
$$

Now calculating the Jacobian matrix $\mathrm{J}$ at $E_{0}$ and then solving $\operatorname{det}(J-\lambda I)$, we get

$$
\left(\delta_{1}+\lambda\right)^{2}\left(\phi+\delta_{1}+\delta_{2}+\lambda\right)\left[(\beta+\lambda)\left(\gamma+\mu+\delta_{1}+\delta_{2}+\lambda\right)-\frac{\alpha \beta \pi}{\delta_{1}}\right]=0
$$

which gives us the eigenvalues, $\lambda_{1}=-\delta_{1}, \lambda_{2}=-\beta, \lambda_{3}=\frac{-B+\sqrt{B^{2}-4 C}}{2}$ and $\lambda_{4}=\frac{-B-\sqrt{B^{2}-4 C}}{2}$, where $B=\beta+\gamma+\mu+\delta_{1}+\delta_{2}$ and $C=\beta\left(\gamma+\mu+\delta_{1}+\delta_{2}-\alpha \pi / \delta_{1}\right)$. Since $\lambda_{1}$ and $\lambda_{2}$ are less than zero, so the DFE is stable when both $\lambda_{3}<0$ and $\lambda_{4}<0$; which satisfied when $C>0$. Therefore, all together we can say that the DFE is stable when $C>0$ and the DFE is unstable when $C<0$.

\subsection{Analysis of EEP}

Again to analyze the endemic equilibrium point [10], let EEP is

$$
E_{1}=(x, y, z, u, v)=\left(\frac{\pi}{\alpha D-\delta_{1}}, \frac{\alpha \pi D}{\beta\left(\alpha D-\delta_{1}\right)}, D, \frac{\gamma D}{\phi+\delta_{1}+\delta_{2}}, \frac{1}{\delta_{1}}\left[\frac{\phi \gamma D}{\phi+\delta_{1}+\delta_{2}}+\mu D\right]\right) .
$$

Then using $x=\frac{\pi}{\alpha D-\delta_{1}}, y=\frac{\alpha \pi D}{\beta\left(\alpha D-\delta_{1}\right)}$ and $z=D$ in the Jacobian $\mathrm{J}$ in (4.1) and then calculating the characteristic equation $\operatorname{det}(J-\lambda I)$ we get

$\left(\phi+\delta_{1}+\delta_{2}+\lambda\right)\left(\delta_{1}+\lambda\right)\left[-\left(\alpha D+\delta_{1}+\lambda\right)(\beta+\lambda)\left(\gamma+\mu+\delta_{1}+\delta_{2}+\lambda\right)+\frac{\alpha \beta \pi}{\alpha D-\delta_{1}}\left(\alpha D+\delta_{1}+\lambda\right)+\frac{\alpha^{2} \beta \pi D}{\alpha D-\delta_{1}}\right]=0$

Solving this we obtain the first two eigenvalues, $\lambda_{1}=-\left(\phi+\delta_{1}+\delta_{2}\right)$ and $\lambda_{2}=-\delta_{1}$ and for the rest of the eigenvalues we have the equation

$$
\lambda^{3}+A \lambda^{2}+B \lambda+C=0
$$


where,

$$
\begin{aligned}
A= & \gamma+\mu+2 \delta_{1}+\delta_{2}+\beta+\alpha D \\
B= & \beta \gamma+\alpha \gamma D+\delta_{1} \gamma+\beta \mu+\alpha \mu D+\delta_{1} \mu+2 \beta \delta_{1}+\alpha D \delta_{1} \\
& +\delta_{1}^{2}+\beta \delta_{2}+\alpha D \delta_{2}+\delta_{1} \delta_{2}+\alpha \beta D-\frac{\alpha \beta \pi}{\alpha D-\delta_{1}} \\
C= & \alpha \beta \gamma D+\beta \gamma \delta_{1}+\alpha \beta \mu D+\beta \mu \delta_{1}+\alpha \beta \delta_{1} D+\beta \delta_{1}^{2} \\
& +\alpha \beta \delta_{2} D+\beta \delta_{1} \delta_{2}-\frac{\alpha^{2} \beta \pi D}{\alpha D-\delta_{1}}-\frac{\alpha \beta \pi \delta_{1}}{\alpha D-\delta_{1}}-\frac{\alpha^{2} \beta \pi D}{\alpha D-\delta_{1}}
\end{aligned}
$$

Since the first two eigenvalues are negative then by applying the Ruth-Harwitz stability criterion [11], we can say that EEP is stable if $A>0, C>0$ and $A B-C>0$.

\subsection{Basic Reproduction Number}

The basic reproduction number $R_{0}$ is the parameter that estimates if a disease spread in the population or it does not. If the parameter $R_{0}<1$ then we can predict that the disease will die out and if $R_{0}=1$ then the disease will stay in the system and it will be stable. But if $R_{0}>1$ then the disease will spread and will cause an outbreak. The larger the value of $R_{0}$, the harder it gets to control it. Now to estimate if COVID-19 will die out or keep spreading in the population, we calculate the basic reproduction number. $R_{0}$ can be calculated by the equation, $R_{0}=\rho\left(F V^{-1}\right)$, that is the spectral radius of $F V^{-1}$ [12]. Hence we have

$$
\begin{gathered}
F=\left[\begin{array}{ll}
0 & \alpha \\
0 & 0
\end{array}\right], \\
V=\left[\begin{array}{cc}
\beta & 0 \\
-\beta & \gamma+\mu+\delta_{1}+\delta_{2}
\end{array}\right] \\
\Rightarrow V^{-1}=\left[\begin{array}{cc}
1 / \beta & 0 \\
\frac{1}{\gamma+\mu+\delta_{1}+\delta_{2}} & \frac{1}{\gamma+\mu+\delta_{1}+\delta_{2}}
\end{array}\right]
\end{gathered}
$$

Then calculating the characteristic polynomial of $F V^{-1}$, we get

$$
\left|F V^{-1}-\omega I\right|=\left|\begin{array}{cc}
\frac{\alpha}{\gamma+\mu+\delta_{1}+\delta_{2}}-\omega & \frac{\alpha}{\gamma+\mu+\delta_{1}+\delta_{2}} \\
0 & 0
\end{array}\right|=0
$$

Therefore, the spectral radius of $F V^{-1}$ is

$$
R_{0}=\rho\left(F V^{-1}\right)=\frac{\alpha}{\gamma+\mu+\delta_{1}+\delta_{2}}
$$

At this point, the stability of the system can be derived by the following theorem.

Theorem 1. [10, 13, 14$]$

(a) If $R_{0}<1$ then the system has positive disease-free equilibria and no endemic equilibria, (b) If $R_{0}>1$ then the system has positive disease-free equilibria and endemic equilibria.

In the following section, we will estimate the parametric values to illustrate the numerical results for further applications. 


\section{$5 \quad$ Numerical Solutions and Applications}

The nonlinear system in (2.2) can be solved using the numerical methods and we can observe the dynamics of the model. To numerically solve the data we estimated the parameters first [4, 14]. The parameters are calculated per 1000/day. The parameters $\alpha, \gamma$ and $\mu$ have been assumed. The total population of the world has been taken, $N=7.57$ billion. The rest of the values are listed below :

\begin{tabular}{|l|l|l|}
\hline Parameter & Value & References \\
\hline$\pi$ & $5.812 \times 10^{-4}$ & {$[15]$} \\
$\alpha$ & $3.11 \times 10^{-1}$ & estimated \\
$\delta_{1}$ & .001 & {$[15]$} \\
$\beta$ & .1735 & {$[4]$} \\
$\gamma$ & .08 & estimated \\
$\delta_{2}$ & $9.7 \times 10^{-3}$ & {$[4]$} \\
$\mu$ & .009 & estimated \\
$\phi$ & $4.87 \times 10^{-4}$ & {$[4]$} \\
$\mathrm{S}(0)$ & 1 & estimated \\
$\mathrm{E}(0)$ & .05 & eatimated \\
$\mathrm{I}(0)$ & .002 & {$[4]$} \\
$I_{s}(0)$ & .0002 & estimates \\
$\mathrm{R}(0)$ & .0003 & {$[4]$} \\
\hline
\end{tabular}

Table 1: Parameters and initial conditions for the World.

Using these parameter values and initial conditions we can solve the system by RungeKutta 4th order method. Here we have used MATLAB to solve the system in (2.2) and then we generated a graph to analyze the dynamics of susceptible, exposed, infected, isolated and recovered population.

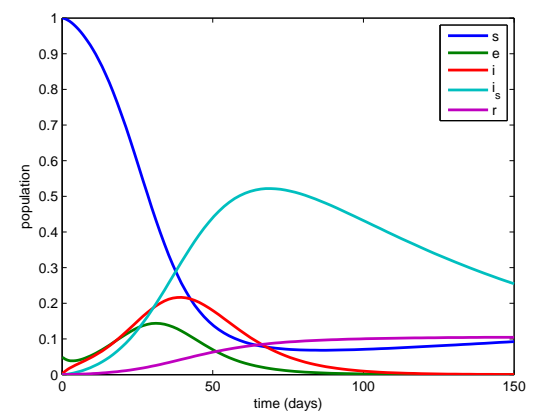

Figure 2: Dynamics of susceptible, exposed, infected, isolated and recovered population over time.

From the Figure 2, we observe that the susceptible population is decreasing with time, 
which means more and more people are getting exposed. Since infected population is increasing, therefore they are getting large number of infected individuals over time which can lead to an outbreak in a very short time. If we analyze the rest of the population dynamics, we see that the infected population grows at a very high rate initially meanwhile the recovered population is not growing as much as infected population which results an outbreak.

Using the parameters that are estimated in Table 1, we can determine the value of the basic reproduction number from (4.2) which is $R_{0}=3>1$, means that if the estimated data stays this way it will create an outbreak. But for calculation we have assumed the value of $\gamma$ and $\mu$ and these may not focus the exact (real) scenario. If we analyze the sensitivity of the model, only then we can conclude how these parameters is going to vary in case of stability of the model.

\subsection{Sensitivity Analysis}

The sensitivity analysis is a very important part in modelling of a infectious disease. It helps us to understand how much a parameter value vary in the disease dynamics. It helps to give us some ideas about what can be done or avoided to prevent the disease. Now we examine the sensitivity analysis of $R_{0}$ with respect to the model parameters [16]. Here we are using the parameter values from Table 1.

We have from equation (4.2), $R_{0}=\alpha /\left(\gamma+\mu+\delta_{1}+\delta_{2}\right)$. Then the sensitivity index of $R_{0}$ with respect to $\alpha$ can be calculated as

$$
\begin{aligned}
\gamma_{\alpha}^{R_{0}} & =\frac{\partial R_{0}}{\alpha} \times \frac{\alpha}{R_{0}} \\
& =\frac{1}{\gamma+\mu+\delta_{1}+\delta_{2}} \times \frac{\alpha\left(\gamma+\mu+\delta_{1}+\delta_{2}\right)}{\alpha} \\
& =1
\end{aligned}
$$

Similarly, we calculate all the sensitivity index:

$$
\begin{aligned}
& \gamma_{\pi}^{R_{0}}=0 . \\
& \gamma_{\delta_{1}}^{R_{0}}=\frac{-\delta_{1}}{\gamma+\mu+\delta_{1}+\delta_{2}}=-0.01 \\
& \gamma_{\beta}^{R_{0}}=0 \\
& \gamma_{\gamma}^{R_{0}}=\frac{-\gamma}{\gamma+\mu+\delta_{1}+\delta_{2}}=-0.8 \\
& \gamma_{\mu}^{R_{0}}=\frac{-\mu}{\gamma+\mu+\delta_{1}+\delta_{2}}=-0.1 . \\
& \gamma_{\delta_{2}}^{R_{0}}=\frac{-\delta_{2}}{\gamma+\mu+\delta_{1}+\delta_{2}}=-0.09 . \\
& \gamma_{\phi}^{R_{0}}=0 .
\end{aligned}
$$

After analyzing the indices we observe the following results: 
1. From $\gamma_{\alpha}^{R_{0}}=1$, we can say that, if the exposure rate $\alpha$ increases $10 \%$, the value of $R_{0}$ increases $10 \%$, which can lead to an outbreak and if $\alpha$ decreases $10 \%$ so does $R_{0}$. So to keep the disease in control, we need to keep the exposure rate low. Which is why the steps like social distancing, home quarantine are being promoted by WHO and governments to control the pandemic.

2. $\gamma_{\gamma}^{R_{0}}$ is negative and from the value we can say increasing the value of $\gamma$ by $10 \%$ we can get $8 \%$ reduction in $R_{0}$. So, we need to isolate the infected person more to stop the disease spread.

3. Similarly from $\gamma_{\mu}^{R_{0}}$ and $\gamma_{\delta_{2}}^{R_{0}}$ we can say increasing the parameters have also a negative effect in $R_{0}$ which means the recovery rate of the non-isolated people and death rate due to the disease need to be increased to avoid outbreak.

4. The value of $\gamma_{\delta_{1}}^{R_{0}}$ is very small. Therefore changes in this parameter does not change $R_{0}$ that much.

\subsection{Case Study: COVID-19 and Bangladesh}

Bangladesh is a south Asian densely populated country where the first cases of coronavirus was reported on 8 March 2020. Since then the country reached to 424 total cases (April 10, 2020). The death rate from infected individuals in Bangladesh is too high, $6.7 \%$ compare to other territories in the Globe on April 10, 2020. With a limited amount of testing kits and treatment facilities this could be a challenge for the country to cope with a pandemic. According the current situation of the country the parameters are estimated in Table 2 and then we have studied the situation according to the model described in (2.2). Although due to the lack of testing centers throughout the country and since the event is going on, the real data may not be available in some cases and so few parametric values are estimated for calculation $[5,17]$. 


\begin{tabular}{|l|l|l|}
\hline Parameter & Value & References \\
\hline$\pi$ & $3.2 \times 10^{-10}$ & {$[5]$} \\
$\alpha$ & 2 & estimated \\
$\delta_{1}$ & 0.0148 & {$[5]$} \\
$\beta$ & 1.02 & {$[17]$} \\
$\gamma$ & .084 & estimated \\
$\delta_{2}$ & $1 \times 10^{-4}$ & {$[17]$} \\
$\mu$ & 0.027 & estimated \\
$\phi$ & $6.6 \times 10^{-6}$ & {$[17]$} \\
$\mathrm{S}(0)$ & 1 & {$[5]$} \\
$\mathrm{E}(0)$ & $1.54 \times 10^{-6}$ & estimated \\
$\mathrm{I}(0)$ & $7.68 \times 10^{-7}$ & {$[17]$} \\
$I_{s}(0)$ & $4.8 \times 10^{-7}$ & estimated \\
$\mathrm{R}(0)$ & $2 \times 10^{-7}$ & {$[17]$} \\
\hline
\end{tabular}

Table 2: Parameters and initial conditions for Bangladesh.

All the parameters are calculated per 1000/day. Using the numerical values of parameters form Table 2, we calculate, $R_{0}=\frac{\alpha}{\gamma+\mu+\delta_{1}+\delta_{2}}=15.88>1$, which means the disease will vastly spread under the current situation and there will be an outbreak. At the next stage, we can observe the dynamics of different population for next few days.

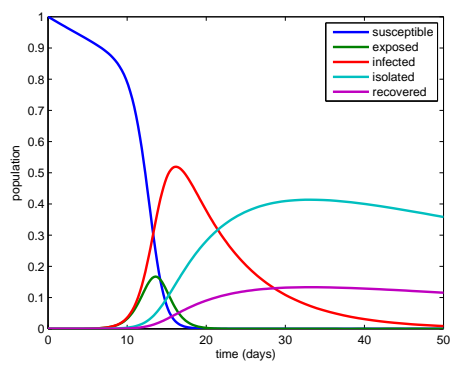

Figure 3: Dynamics of susceptible, exposed, infected, isolated and recovered population over time.

From Figure 3, we can say the virus will transmit rapidly over time resulting a vastly infected population and the situation may even get out of control if no necessary steps are taken. Therefore, it is high time to take precautions to minimize the damage.

From the sensitivity analysis with the global data, we observe that decreasing the virus transmission rate helps to control the system as well as increasing isolation rate and recovery rate of the non-isolated people also some of the important cases to consider. To control the public mobility, the government has already imposed lockdown from 24 March till 25 April (2020) all over the country to maintain the social distance whereas the pharmacies will remain open including necessary food stores. People are asked to stay 
at home, all social and religious events are said to be celebrated in niche to avoid public gathering. The Figure 4 illustrates the effectiveness of lockdown for 20 or more days using some roughly assumed calculations and possibilities [9].

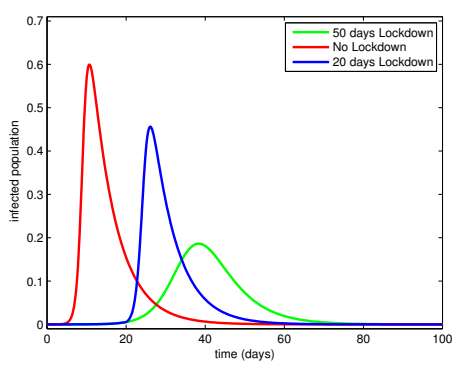

Figure 4: Dynamics of infected population with and without lockdown.

We assume that, with no lockdown $75 \%$ and with lockdown $45 \%$ of the population is socially active and getting exposed. Then the exposure rate, $\alpha=2 \times 0.75=1.5$, with no lockdown and $\alpha=2 \times 0.45=0.9$ with lockdown. From the Figure 4 , the result is seen clearly. It reduces the infected population drastically which means social distancing is a major key to face this epidemic in this scenario. Only 20 days lockdown is not sufficient to control the epidemic and huge number of population will be infected while if we consider 50-60 days lockdown it shows that the pandemic can be under control with lowest number of infections and death toll, see Figure 4 for details. The visible curves on the diagram 4 shows the approximate results; although we assume that there are many unreported data due to the lack of healthcare system of Bangladesh.

Again, we consider the step where infected patients get more isolated. First we assume that if $70 \%$ more infected population is getting isolated, we study the effect from the graph for $\gamma=0.084, \gamma=.084+0.7 \times 0.084=0.1428$ and sequentially some other values of $\gamma$.

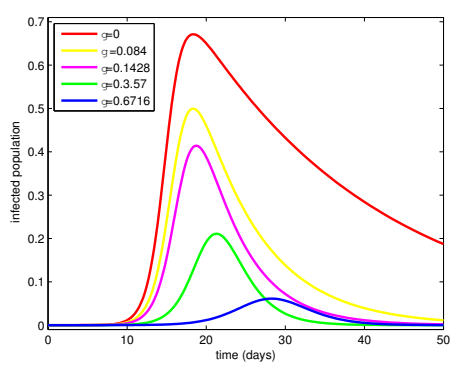

Figure 5: Dynamics of infected population with and without more isolation.

We observe that the infected population is slowing down with more isolation rate, see Figure 5. Increasing value of $\gamma$ ensures the long run social isolation and physical distance which imply to reduce infection and death toll as seen in Figure 5. Therefore, we can conclude that decreasing the exposure rate by socially distancing the population 
and increasing the isolation rate of the infected population both can be necessary steps to control the epidemic.

\section{Summary and Conclusion}

To estimate the situation of global pandemic COVID-19, epidemic modelling is a very effective way if the parameters can be estimated properly. We have modified the SEIR model into a $\mathrm{SEII}_{s} \mathrm{R}$ model where we have considered the isolation process of the infected patients. The parameters of the model are estimated by collecting the most recent data the COVID-19 pandemic of the world and some parameters were assumed since the real data are not available. Then we calculate the basic reproduction number to examine the stability of the system and we had $R_{0}=3$ from Table 1 , which means the system is not stable. Meanwhile we have also estimated the values for Bangladesh in Table 2 and from these values we observed $R_{0}>1$ and there will be an outbreak under current situation. But while we are studying this cases the data is changing every hour. Since it is an ongoing process with fluctuations the real parameter and real value of $R_{0}$ may be different from what we have calculated which is a limitations in this study.

The sensitivity of $R_{0}$ with respect to all the parameters have been analyzed and we have drawn some results regarding the calculations. Since the infected individuals cannot easily be identified because the virus is reported to stay in incubation period for a long time which results an asymptomatic patient and the virus is known to be powerful enough to transmit even in the incubation period. Therefore the best solution of the situation can only be a lockdown situation where everyone can be kept distant from infected individuals. We have also seen that the increment of rate of isolation and the rate of recovery for nonisolated patients can also help to keep the situation in control. Finally, we have discussed graphically how lockdown in Bangladesh for next 20 days or more and isolating the patients are significantly important to control the COVID-19 situation.

\section{References}

[1] https://www.who.int/emergencies/diseases/novel-coronavirus-2019

[2] Alam MS, Alam MZ, Nazir KHMNH and Bhuiyan MAB. The emergence of Novel Coronavirus (COVID-19) in Bangladesh: Present Status, Challenges, and Future Management. Journal of Advanced Veterinary and Animal Research. 2020; 7(2): 198-208.

[3] Kamrujjaman M, Mahmud MS and Islam MS. Coronavirus Outbreak and the Mathematical Growth Map of COVID-19. Annual Research \& Review in Biology. 2020; 35(1): $72-78$.

[4] https://www.worldometers.info/coronavirus/

[5] https://en.wikipedia.org/wiki/Demographics_of_Bangladesh 
[6] Kermack WO and McKendrick AG. A Contribution to the Mathematical Theory of Epidemics. Proceedings of the Royal Society. 1927; 115(772): 700-722.

[7] Castillo-Chavez C and Feng Z. Mathematical models for the disease dynamics of tuberculosis. Advances in Mathematical Population Dynamics-Molecules, Cells and Man. 1996; 1-28.

[8] Alam M, Kabir KMA and Tanimoto J. Based on Mathematical Epidemiology and Evolutionary Game Theory, Which Is More Effective: Quarantine or Isolation Policy? Journal of Statistical Mechanics Theory and Experiment. 2020; 2020(3): 033502.

[9] Chowdhury A, Kabir KMA and Tanimoto J. How Quarantine and Social Distancing Policy Can Suppress the Outbreak of Novel Coronavirus in Developing or Under Poverty Level Countries: A Mathematical and Statistical Analysis. 2020; preprint.

[10] Affi PO. Sensitivity Analysis of the SEIR Epidemic Compartmental Model. International Journal of Science and Research. 2018; 352-357.

[11] DeJesus EX and Kaufman C, Routh-Hurwitz criterion in the examination of eigenvalues of a system of nonlinear ordinary differential equations. American Physical Society. 1987.

[12] Diekmann O, Heesterbeek JAP and Roberts MG. The Construction of NextGeneration Matrices for Compartmental Epidemic Models. Journal of The Royal Society Interface. 2009; 7(47): 873-885.

[13] Yang C and Wang J. A Mathematical model for the novel coronavirus epidemic in Wuhan, China. AIMS Press. 2020.

[14] Victor AO, Mathematical Predictions for COVID-19 as a Global Pandemic. 2020; medRxiv preprint.

[15] https://worldpopulationreview.com/

[16] Olaniyi S, Lawal MA and Obabiyi OS. Stability and Sensitivity Analysis of a Deterministic Epidemiology Model with Pseudo-Recovery. IAENG International Journal of Applied Mathematics. 2016; 46(2): 1-8.

[17] https://www.iedcr.gov.bd/

\section{Declarations}

Ethics approval and consent to participate

The ethical approval or individual consent was not applicable.

Availability of data and materials

All data and materials used in this work were publicly available.

\section{Consent for publication}


Not applicable.

Funding

None.

\section{Acknowledgements}

None.

\section{Disclaimer}

None.

\section{Competing Interests}

Authors have declared that no competing interests exist.

\section{Authors' Contributions}

MSI and JII derived the mathematical model, designed the study and the first draft of the manuscript. JII, AK and MK carried out the data analysis, some numerical simulations and the parameter estimations. MSI, JII and MK provided the literature review and final drafting. All authors contributed to the reviewing of the manuscript. All authors read and approved the final manuscript. 